die amerikanische Zulassungsbehörde hat bereits die Registerverantwortlichen eingeladen, um den Nutzen der katheterbasierten Techniken besser bewerten zu können.

Wann operiert oder kathetert werden sollte, ist eine klinische, fallbezogene Entscheidung, die im HerzTeam diskutiert und dann dem Patienten vorgeschlagen werden sollte. Für die Risikoabschätzung werden der STS-Score und der EuroScore-II verwendet, neuerdings zunehmend auch der deutsche AKL-Score. Diese Scores erfassen wichtige Komorbiditäten wie eine reduzierte Auswurffraktion, periphere Verschlusskrankheit, Gebrechlichkeit (frailty), Porzellanaorta. Sie versuchen, möglichst genaue prognostische Abschätzungen zu liefern.

Wohingegen für Patienten mit hohem Risiko die transvaskulären Verfahren (TAVI) indiziert sind, sind Fälle mit intermediärem Risiko Kandidaten für beide Optionen. Die Entscheidung sollte dann im Herz-Team sorgfältig abgewogen werden.

\section{TAVI keine Goldader}

Ökonomische Gesichtspunkte sollten dabei keinen Ausschlag geben. Die DRG-Ziffer F98Z sieht eine Honorierung für die „Endovaskuläre Implantation eines Herzklappenersatzes oder transapikaler Aortenklappenersatz" von etwa $€ 33000$ vor, wobei bereits für die

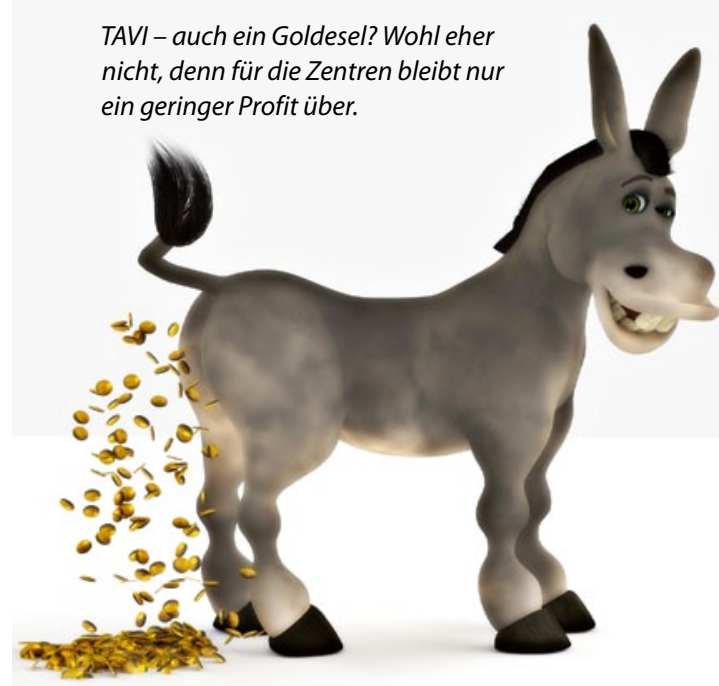

Klappen-Systeme jeweils zwischen $€ 15000$ bis 20000 entfallen, wie Walther vorrechnete. Für die Hospitäler bleibe bei Routinefällen nur ein geringer Profit über. Anders sehe es aus, wenn es zu komplizierten Verläufen mit mehrtägigem Aufenthalt auf der Intensivsta- tion und evtl. Dialyse kommt, dann fällt die Kostenrechnung defizitär aus. Von einem Goldesel für die Zentren könne also im Zusammenhang mit dem TAVI nicht die Rede sein. Zusammenfassend betonten Walther und sein interventioneller Kollege R. Bekeredjian aus Heidelberg, dass es darum gehen müsse, den individuell am besten passenden Aortenersatz zu finden, und zwar für die klassischen Routinefälle genauso wie für Patienten mit intermediärem oder hohem Risiko. Der Sicherheitsaspekt müsse dominieren. Verbesserungen der Devices hinsichtlich ihrer Steuerbarkeit sind in Arbeit, die Einpassgenauigkeit, vor allem die Vermeidung von paravalvulären Lücken, ist für den Erfolg ebenso entscheidend wie die Berücksichtigung des Verkalkungsgrads der Klappe und der Komorbiditäten. Über die Langlebigkeit der TAVI könne man erst in den nächsten Jahren verlässliche Auskünfte geben, die Auswertung der GARY-

Registerdaten dürfte dabei hilfreich sein.

Und: In die Entscheidungen der HerzTeams, das forderten zahlreiche Diskutanten, sollten unbedingt auch Fragen der Lebensqualität der Patienten einfließen.

Dr. med. Jochen Aumiller I!

\title{
GARY-Register: Erste Zwischenbilanz mit den 1-Jahres-Daten
}

Den aktuellen Stellenwert von TAVI machten die Daten des deutschen Aortenklappen-Registers (German Aortic Valve RegistrY - GARY) deutlich. Prof. Christian Hamm, Gießen und Bad Nauheim, stellte sie vor. Die 1-Jahres-Daten sind, so Hamm, außerordentlich erfreulich. Wichtigstes Ergebnis: Patienten mit einem hohen Risiko können mit der TAVI mindestens mit gleichem Erfolg wie mit einer konventionellen Operation behandelt werden, mit dem Vorteil, dass TAVI der schonendere Eingriff ist.

Hier die Details: 13860 Patienten haben im Jahr 2011 in Deutschland eine neue Aortenklappe erhalten, 6523 einen offenen chirurgischen Aortenklappenersatz, bei weiteren 3462 Patienten wurde zusätzlich mindestens ein Bypass gelegt.
Der interventionelle Arm des Registers: 2694 transvaskuläre TAVIs und 1181 transapikale TAVIs. Eine Auswertung der Krankenhausmortalität zeigte für die TAVI eine Sterberate von 5,5\% (transvaskulär) bzw. 7,8\% (transapikal). Während oder unmittelbar nach einem offenen Aortenklappenersatz starben nur 2,2\% der Patienten.

Werden die Daten in den Hochrisikogruppen verglichen, so findet sich bei der TAVI im Vergleich zur konventionellen Operation kein erhöhtes Sterberisiko mehr. Auch die höhere Sterblichkeit nach transapikaler TAVI lässt sich dadurch erklären, dass diese hauptsächlich bei sehr kranken Patienten mit schlechtem Gefäßzustand zum Einsatz kommt. Bei der überwiegenden Mehrzahl der Patienten
$(98,1 \%)$ war eine Beobachtung über ein Jahr möglich. Nach diesem Jahr waren noch fast 12000 der Patienten am Leben. In allen Patientengruppen nahm die Sterblichkeit zu. Nach offener Klappenimplantation war die Gesamtsterblichkeit am geringsten, gefolgt von der offenen Klappenimplantation mit Bypass, transvaskulärer TAVI und transapikaler TAVI. In den TAVI-Gruppen lag die Mortalität nach einem Jahr über $20 \%$. Wie Hamm betonte, müssen in die Bewertung dieser Daten die Ausgangsbedingungen mit einbezogen werden: So waren mehr als $80 \%$ der TAVI-Patienten älter als 75 Jahre und im Durchschnitt deutlich kränker. Für die meisten von ihnen war TAVI wohl die letzte Chance.

(J.A.) II 